\title{
Caracterização de cepas de Schistosoma mansoni por morfometria de vemes adultos provenientes de infecção unissexual
}

\author{
Characterization of Schistosoma mansoni strains by morphometry \\ of adult worms derived from single-sex infection
}

\author{
José Roberto Machado-Silva ${ }^{1}$, Renata Heisler Neves ${ }^{1}$, Luciana Ormond ${ }^{1}$, \\ Maarten Hulstijn ${ }^{1}$ e Delir Corrêa Gomes ${ }^{2}$
}

\begin{abstract}
Resumo Camundongos foram infectados com cercárias, de um único sexo, de cepas simpátricas do Schistosoma mansoni. Nos vermes adultos, foram encontradas diferenças significativas $(p<0,05)$ nas ventosas, lobos testiculares, ovário e espessura do tegumento. O experimento demonstra que a morfometria de vermes isolados de infecção unissexual também é uma ferramenta na identificação de cepas do Schistosoma mansoni.
\end{abstract}

Palavras-chaves: Schistosoma mansoni. Cepas. Infecção unissexual. Estudo morfométrico.

Abstract Mice were infected with only one sex cercaria derived from sympatric strains of Schistosoma mansoni. Adult worms presented significative differences $(p<0.05)$ regarding suckers, testicular lobes, ovary and thickness of the tegument. Data show that morphometric study of unisexual infection worms can be also used for characterization of Schistosoma mansoni strains.

Key-words: Schistosoma mansoni. Strain. Unisexual infection. Morphometric study.

Desde longa data, as infecções unissexuais por Schistosoma mansoni tem sido estudadas com diferentes abordagens. Nas áreas de baixa intensidade de transmissão da esquistossomose mansoni, os moluscos são parasitados geralmente por um único sexo do parasito ${ }^{16}$. Ainda que não seja freqüente o encontro de vertebrados naturalmente infectados com infecção unissexual, há uma evidente maior ocorrência de vermes adultos do sexo masculino ${ }^{520}$, o que sugere que as cercárias machos apresentem uma maior capacidade infectante $^{3}$ ou número maior no meio aquático.

A identificação do sexo das formas larvárias do S. mansoni por características fenotípicas tem sido questionada ${ }^{17}$. Entretanto, o sexo das cercárias foi determinado por análise morfométrica computadorizada do corpo e cauda $^{7}$ e pelo padrão de papilas argentofílicas $^{6}{ }^{16} 18$. Diversos autores empregaram a morfometria de vermes adultos em infecções mistas para caracterizar cepas de S. mansonit 79101321.
Entretanto, não é conhecido se a identificação de cepas pode também ser feita a partir de vermes provenientes de infecção unissexual. Neste artigo, pela primeira vez, esta hipótese foi testada e confirmada.

Espécimes de Biomphalaria glabrata, simpátrica, foram infectados por um único miracídio isolado das fezes do roedor silvestre Nectomys squamipes (Muridae: Sigmondontinae) ou de casos humanos procedentes do município de Sumidouro, no estado do Rio de Janeiro ${ }^{6}$. A cepa de roedor foi identificada como SR (Sumidouro-roedor), enquanto a humana foi denominada $\mathrm{SH}$ (Sumidouro-humana).

Após a exposição dos moluscos a um foco luminoso $^{1}, 50$ cercárias eliminadas foram empregadas para infectar Mus musculus Swiss Webster, por via transcutânea. Após a infecção, os animais foram mantidos em gaiolas de polipropileno $(40 \mathrm{~cm} x$ $33 \mathrm{~cm}$ ) e alimentados com a ração industrializada convencional Nuvilab CR1 (município de Colombo,

\footnotetext{
1. Disciplina de Parasitologia do Departamento de Patologia e Laboratórios da Faculdade de Ciências Médicas da Universidade do Estado do Rio de Janeiro, Rio de Janeiro, RJ. 2. Laboratório de Helmintos Parasitos de Vertebrados do Departamento de Helmintologia do Instituto Oswaldo Cruz da Fundação Oswaldo Cruz, Rio de Janeiro, RJ. Apoio: UERJ, CNPq

Endereço para correspondência: Dr. José Roberto Machado e Silva. Disc. de Parasitologia/Dept ${ }^{\circ}$ de de Patologia e Laboratórios/FCM/UERJ. R. Prof. Manuel de Abreu 444/5 andar, Vila Isabel, 20551-170 Rio de Janeiro, RJ, Brasil.

Telefax: 5521 2587-6148.

e-mail:machado@uerj.br

Recebido para publicação em 26/2/2002

Aceito em 4/12/2003
} 
Estado do Paraná, Brasil) e água ad libitum. Os experimentos foram realizados de acordo com os procedimentos éticos com animais de experimentação ${ }^{23}$.

Após 48 dias os animais foram mortos, perfundidos com salina citratada a $0,9 \%^{22}$ e os vermes adultos recolhidos dos vasos intra-hepáticos ou da veia porta. Após a fixação em AFA (ácido acético, formalina e álcool $95 \%$ ), os espécimes foram corados por carmim clorídrico, montados em lâminas histológicas e analisados por morfometria em um software de análise de imagens (IMAGE PRO PLUS, USA).

Os seguintes caracteres foram avaliados: área da ventosa oral e ventral, distância entre elas e espessura do tegumento (em ambos os sexos), número, área, perímetro e maior/menor diâmetro dos lobos

Tabela 1 - Dados morfométricos dos vermes adultos machos.

\begin{tabular}{lrr}
\hline & \multicolumn{2}{c}{ Cepas } \\
\cline { 2 - 3 } Caracteres morfológicos & $\mathrm{SH}(\mathrm{X} \pm \mathrm{DP})$ & $\mathrm{SR}(\mathrm{X} \pm \mathrm{DP})$ \\
\hline Lobos testiculares & $20128 \pm 3540$ & $26972 \pm 5413$ \\
$\quad$ área $\left(\mu \mathrm{m}^{2}\right)^{*}$ & $254 \pm 40$ & $382 \pm 65$ \\
maior diâmetro $(\mu \mathrm{m})^{*}$ & $76 \pm 12$ & $59 \pm 19$ \\
menor diâmetro $(\mu \mathrm{m})^{*}$ & $759 \pm 120$ & $1220 \pm 246$ \\
$\quad$ perímetro $(\mu \mathrm{m})^{*}$ & $7 \pm 1$ & $8 \pm 1$ \\
número $(\mu \mathrm{m})^{*}$ & & $22212 \pm 5554$ \\
Ventosas & $18433 \pm 3388$ & $281 \pm 74$ \\
$\quad$ área da ventosa oral $\left(\mu \mathrm{m}^{2}\right)^{*}$ & $18426 \pm 6480$ & $28629 \pm 7356$ \\
$\quad$ área da ventosa ventral $\left(\mu \mathrm{m}^{2}\right)^{*}$ & & $10 \pm 4$ \\
$\quad$ distância entre elas $(\mu \mathrm{m})^{*}$ & $10 \pm 3$ & \\
Tegumento & & \\
$\quad$ espessura $(\mu \mathrm{m})$ & & \\
* diferença significativa $\mathrm{p}<0,05$ & & \\
& & \\
& &
\end{tabular}

Atualmente, a aplicação de técnicas de biologia molecular como a reação em cadeia da polimerase (PCR) tem sido uma importante ferramenta para a identificação de cepas do Schistosoma mansoni e o sexo das cercárias $^{2}$. Entretanto, o seu acesso pode ser restrito devido à necessidade de equipamentos especializados ${ }^{17}$. Neste caso, a infecção experimental de camundongos é uma estratégia amplamente utilizada que atende a parte destas dificuldades. Evidentemente, este procedimento é demorado, de aplicação limitada, quando a situação requer imediata identificação da cepa/sexo do parasito ${ }^{7}$. Apesar desta limitação, consideramos que é uma metodologia necessária para a obtenção de vermes adultos que após corados e analisados por morfometria, tornam-se uma ferramenta útil para a identificação de cepas alopátricas do Schistosoma mansoni 491421. testiculares; área, perímetro, comprimento e largura do ovário ${ }^{10} 11^{12} 13$. Para a cepa de roedor (SR) foram estudados 77 espécimes (62 machos e 15 fêmeas) e 85 (61 machos e 24 fêmeas) na cepa humana ( $\mathrm{SH}$ ).

Os valores encontrados foram analisados estatisticamente pelo teste $t$ de Student, considerando diferença significativa $p<0,05$.

Os valores morfométricos dos vermes adultos machos estão apresentados na Tabela 1. Todos os caracteres foram considerados como diferenças significativas $(p<0,05)$ exceto a espessura do tegumento. Encontramos apenas um lobo testicular supranumerário nos espécimes estudados na cepa $\mathrm{SH}$. Nas fêmeas as diferenças estavam, apenas, no perímetro do ovário e espessura do tegumento (Tabela 2).

Tabela 2 - Dados morfométricos dos vermes adultos fêmeas.

\begin{tabular}{lrr}
\hline & \multicolumn{2}{c}{ Cepas } \\
\cline { 2 - 3 } Caracteres Morfológicos & $\mathrm{SH}(\mathrm{X} \pm \mathrm{DP})$ & $\mathrm{SR}(\mathrm{X} \pm \mathrm{DP})$ \\
\hline Ovário & $4190 \pm 2149$ & $5646 \pm 1275$ \\
$\quad$ área $\left(\mu \mathrm{m}^{2}\right)$ & $325 \pm 87$ & $392 \pm 64$ \\
$\quad$ comprimento $(\mu \mathrm{m})$ & $36 \pm 8$ & $37 \pm 4$ \\
$\quad$ largura $(\mu \mathrm{m})$ & $118 \pm 36$ & $145 \pm 14$ \\
$\quad$ perímetro $(\mu \mathrm{m})^{*}$ & & \\
Ventosas & $3293 \pm 1995$ & $2877 \pm 450$ \\
$\quad$ área da ventosa oral $\left(\mu \mathrm{m}^{2}\right)$ & $2686 \pm 1741$ & $2195 \pm 518$ \\
$\quad$ área da ventosa ventral $\left(\mu \mathrm{m}^{2}\right)$ & $136 \pm 40$ & $149 \pm 30$ \\
$\quad$ distância entre elas $(\mu \mathrm{m})$ & & \\
Tegumento & \multicolumn{2}{c}{$4 \pm 1$} \\
$\quad$ espessura $(\mu \mathrm{m})^{*}$ & & $5 \pm 1$ \\
\hline * diferença significativa $\mathrm{p}<0,05$ & &
\end{tabular}

Em publicações prévias realizadas com as mesmas cepas do presente estudo, verificamos que os vermes adultos eram morfométricamente diferentes ${ }^{8}{ }^{13}$ embora, não tenham sido evidenciadas diferenças em cercárias ${ }^{6}$. Ainda que possa ser considerado que a mudança de hospedeiro induz a modificações morfológicas nos vermes adultos (plasticidade fenotípica) ${ }^{11}$, o camundongo deve ter exercido a mesma pressão seletiva já que as cepas eram simpátricas porém, circulantes em distintos hospedeiros ${ }^{19}$.

Em síntese, neste experimento foi demonstrado, pela primeira vez, que a morfometria de vermes isolados de infecções por um único sexo em camundongos também é uma ferramenta para a identificação de cepas de Schistosoma mansoni.

\section{REFERÊNCIAS}

1. Bogéa T, Favre TC, Rotenberg L, Silva HS, Pieri OS. Circadian pattern of cercarial emergence in Schistosoma mansoni (Plathyhelminthes: Digenea) from isolated Biomphalaria glabrata. Chronobiology International 13: 93-101, 1996.

2. Boissier J, Durand P, Moné H. PCR effectiveness for sexing Schistosoma mansoni cercariae: application for sexing clonal cercariae populations. Molecular Biochemical and Parasitology 112: 139-141, 2001.

3. Boissier J, Morand S, Moné H. A review of performance and pathogenecity of male and female Schistosoma mansoni during the life-cycle. Parasitology 119: 447-454, 1999. 
4. Coles GC, Thruston JP. Testes number in east african Schistosoma mansoni. Journal of Helminthology 44: 69-73, 1970.

5. D'Andrea OS, Maroja LS, Gentile R, Cerqueira R, Maldonado Jr A, Rey L. The parasitism of Schistosoma mansoni (DigeneaTrematoda) in a naturally infected population of rats, Nectomys squamipes (Rodentia-Sigmodontinae) in Brazil. Parasitology 120: 573-582, 2000.

6. Freire N, Machado-Silva JR, Rodrigues-Silva R, Rey L. Cercarial chaetotaxy and sex determination of Schistosoma mansoni deriving from humans and Nectomys squamipes (Muridae: Sigmondontinae) in Brazil. Memórias do Instituto Oswaldo Cruz 97: 127-132, 2002.

7. Machado-Silva JR, Galvão C, Oliveira RMF, Presgrave OAF, Gomes DC. Schistosoma mansoni Sambon, 1907: comparative morphological studies of some Brazilian strains. Revista do Instituto de Medicina Tropical de São Paulo 37:441-443, 1995.

8. Machado-Silva JR, Galvão C, Presgrave OA, Rey L, Gomes DC. Host-induced morphological changes of Schistosoma mansoni Sambon, 1907 male worms. Memórias do Instituto Oswaldo Cruz 89: 411-416, 1994.

9. Machado-Silva JR, Silva CH, Pereira MJS, Pinto RM, Oliveira RMF, Gomes DC. Evaluation of differences in brazilian strains of Schistosoma mansoni cercariae of both sexes by means of morphometrics analysis. Memórias do Instituto Oswaldo Cruz 95: 839-842, 2000.

10. Magalhães LA, Carvalho JF. Estudo morfológico de Schistosoma mansoni pertencentes a linhagens de Belo Horizonte (MG) e de São José dos Campos (SP). Revista de Saúde Pública de São Paulo 7:289-294, 1973.

11. Neves RH. Aplicação de técnicas morfológicas (microscopia de luz e microscopia de varredura laser confocal) no estudo morfométrico da plasticidade fenotípica em vermes adultos machos e fêmeas de Schistosoma mansoni Sambon, 1907. Tese de Mestrado, Universidade do Estado do Rio de Janeiro, RJ, 2001

12. Neves RH, Machado-Silva JR, Pelajo-Machado M, Oliveira SA, Coutinho EM, Lenzi HL, Gomes DC. Morphological aspects of Schistosoma mansoni adult worms isolated from nourished and undernourished mice: a comparative analysis by confocal laser scanning microscopy. Memórias do Instituto Oswaldo Cruz 96: 1013-1016, 2001.
13. Neves RH, Oliveira AS, Machado-Silva JR, Coutinho EM, Lenzi HL, Gomes DC. Phenotypic characterization of Schistosoma mansoni adult worms recovered from undernourished mice: a morphometric study focusing on the reproductive system. Revista da Sociedade Brasileira de Medicina Tropical 35: 405-407, 2002.

14. Neves RH, Pereira MJS, Gomes DC, Oliveira RMF, MachadoSilva JR. Morphometric differences of adult worms from sympatric samples of Schistosoma mansoni Sambon, 1907 isolated from rodents and humans. Memórias do Instituto Oswaldo Cruz 93: 309-312, 1998.

15. Paraense WL, Corrêa LR. Observations on two biological races of Schistosoma mansoni. Memórias do Instituto Oswaldo Cruz 76: 287-291, 1981.

16. Paraense WL, Santos JM. O sexo do Schistosoma mansoni em infestações produzidas por cercárias de um único molusco. Memórias do Instituto Oswaldo Cruz 47: 35-49, 1949.

17. Pino LA, Morales G, Matinella L. Quetotaxia cercariana de cepas venezolanas de Schistosoma mansoni. Boletin Chileno de Parasitologia 52: 61-66, 1997.

18. Pino LA, Morales G, Noya BA, Noya A. La quetotaxia cercariana en la diferenciación de sexos de Schistosoma mansoni. Menórias do Instituto Oswaldo Cruz 83: 367-374, 1988.

19. Quack T, Doenhoff M, Kunz W, Grevelding CG. Schistosoma mansoni: The varying occurrence of repetitive elements in different strains shows sex-specific polymorphisms. Experimental Parasitology 89: 222-227, 1998.

20. Rodrigues-Silva R, Machado-Silva JR, Faerstein NF, Lenzi HL, Rey L. Natural infection of wild rodents by Schistosoma mansoni. Parasitological aspects. Memórias do Instituto Oswaldo Cruz 87: 271-276, 1992.

21. Saoud MFA. Comparative studies on the characteristics of some geographical strains of Schistosoma mansoni in mice and hamsters. Journal of Helminthology 39: 101-112, 1965.

22. Smithers SR, Terry RJ. The infection of laboratory hosts with cercariae of Schistosoma mansoni and the recovery of the adults worms. Parasitology 55: 695-700, 1965.

23. Working Committee for the Biological Characterization of Laboratory Animals/GV-SOLAS. Guidelines for specification of animals and husbandry methods when reporting the results of animal experiments. Laboratory Animals 19: 106-108, 1985. 